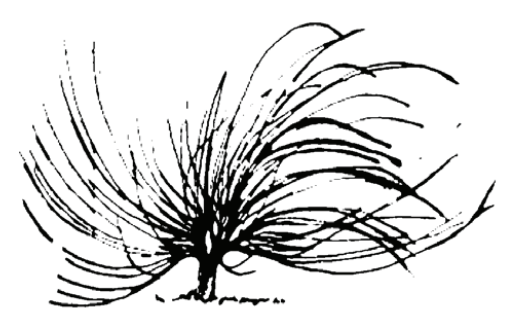

\title{
Afinidad Tecnológica del Profesor Universitario
}

\author{
Ana Teresa Morales Rodríguez ${ }^{1}$ \\ Universidad Veracruzana \\ Xalapa Ver., México \\ ateremora@gmail.com \\ Alberto Ramírez Martinell ${ }^{2}$ \\ Universidad Veracruzana \\ Xalapa Ver., México \\ albramirez@uv.mx
}

\begin{abstract}
Resumen
La afinidad tecnológica de los profesores es uno de los factores identificados como determinante para la integración de las Tecnologías de la Información y la Comunicación (TIC) dentro del contexto académico. En este artículo se cuenta del primer acercamiento a la afinidad tecnológica de profesores de Informática, en el marco del proyecto de Brecha Digital, de la Universidad Veracruzana (UV), de la cual descubrimos que los profesores poseen una afinidad tecnológica común -en términos de Bourdieu, un habitus tecnológico, que en este caso se caracteriza por creencias positivas acerca del uso de las TIC en las funciones académicas de investigación, docencia, gestión y vinculación; representaciones de la computadora, laptop e Internet como recursos indispensables en el contexto de los profesores;
\end{abstract}

(2) $(\theta \Theta$

Recibido: 5 de diciembre de 2014 - Aprobado: 18 de agosto de 2015

1 Estudiante de Doctorado en Investigación Educativa, Universidad Veracruzana; Maestría en Computación Aplicada, Laboratorio Nacional de Informática Avanzada.

2 Profesor-Investigador del Instituto de Investigaciones en Educación de la Universidad Veracruzana; Doctor en Tecnología Educativa, Universidad de Lancaster Inglaterra. 
sin embargo, al interior se cuenta con percepciones que fragmentan el habitus, como la falta de valoración acerca del uso de dispositivos móviles y otras reservas respecto al uso de las TIC en el contexto académico.

Palabras clave: afinidad tecnológica, percepciones, profesor, educación superior, TIC

\begin{abstract}
Technological affinity of faculty members of Higher Education (HE) is a determinant factor for ICT integration in HE institutions. This paper presents a first approach to technological affinity of faculty members of computer science at "Universidad Veracruzana," which is part of a project called Digital Divide into Universidad Veracruzana (UV), and we found common perceptions among the academic community -that we can understand as a collective habitus, in terms of Bourdieu-. This habitus, in the case of faculty members of computer science is characterized by positive beliefs about the use of ICT in the academic functions such as research, teaching, management; perceptions about computer, laptop and Internet as key resources into their academic context; however, some perceptions of teachers fragment the habitus, as the lack of consideration on the use of mobile devices and other reservations about the use of ICT's in the academic context.
\end{abstract}

Keywords: technological affinity, perceptions, teachers, higher education, ICT

\title{
Introducción
}

Este artículo forma parte de una investigación que estudia cómo los profesores universitarios -de cuatro licenciaturas de la Universidad Veracruzana, región Xalapa, Veracruz, México- integran las Tecnologías de Información y Comunicación (TIC) en sus funciones académicas $^{3}$. En este estudio, se han seleccionado cuatro licenciaturas

3 Tesis de Doctorado de Ana Teresa Morales Rodríguez, adscrita al Macro Proyecto "Brecha Digital de los actores universitarios". www.uv.mx/blogs/brechadigital/. 
según la taxonomía de Tony Becher (2001), sin embargo en este texto únicamente se presenta la afinidad tecnológica de los profesores de la licenciatura en Informática (Dura-Aplicada) que junto con las licenciaturas en Filosofia (Blanda-Pura), Idiomas (Blanda-Aplicada) y Biología (Dura-Pura) satisfacen dicha categorización disciplinar. Con respecto a las dimensiones de análisis, vale la pena aclarar que en este artículo solamente se aborda la afinidad -o percepción- como dimensión de análisis, dejando a elementos como el acceso a recursos digitales, saberes digitales del profesor, el grado de apropiación tecnológica, y capital para otras comunicaciones.

La facultad de Informática es una dependencia académica con una población aparentemente afín a las TIC, ya que estas son su objeto de estudio; sin embargo, esto no implica que la tecnología digital se haya incorporado en sus funciones académicas por lo que decidimos explorar mediante una encuesta, la percepción, disposición o afinidad tecnológica de los profesores de Informática.

Para observar la afinidad tecnológica, se han utilizado los indicadores definidos en el marco del proyecto de Brecha digital-UV, que proporcionan información acerca de sus creencias respecto del impacto de las TIC; el uso de recursos digitales (computadora, laptop, tableta y smartphone) y acceso a Internet con fines académicos; además de su percepción acerca de si estas facilitan el establecimiento de redes académicas, si son fuente importante de información y si estar actualizado en tecnología es relevante para su desempeño académico.

El estudio de las percepciones de los profesores universitarios no es empresa fácil, ya que representa por un lado, un reto logístico en lo que respecta al acercamiento a los informantes, por otro, es complejo objetivar y presentar en términos cuantificables un concepto subjetivo como la percepción, ya que los informantes pueden verse influenciados por la figura de la encuesta como un medio de evaluación condicionando así sus respuestas en función del deber ser y no de lo que realmente es.

\section{Marco Teórico}

Para encuadrar el presente problema de estudio, se ha realizado una breve revisión de literatura sobre la percepción de los profesores universitarios acerca de integración de las TIC en sus prácticas académicas; además de una reflexión -orientada a partir de autores clave- sobre 
la integración de las TIC y cómo en cada disciplina se presentan diferentes creencias y disposiciones respecto de la tecnología digital.

\section{La percepción de las TIC del profesor universitario}

Gulbahar y Guven (2008) aseguran que la percepción de las TIC ha sido reconocida universalmente como un factor importante en el éxito de la integración tecnológica dentro de la Educación (p. 43), aseveración apoyada por otros autores como Georgina y Olson (2007) que retoman la idea de Zhoa y Cziko (2001) quienes demuestran -en ambos estudios- que la percepción del profesor acerca de la efectividad de la tecnología determina si la utilizará o no. Por su parte, Zare-ee (2007) encontró que la integración tecnológica de los profesores universitarios se puede ver afectada por percepciones socioculturales limitadas, además considera que los puntos de vista y percepciones de los profesores determinan cómo utilizan las TIC y si se emplean sus potencialidades de manera eficaz para la enseñanza e investigación (p. 320).

Por su parte, Ouzts y Palombo (2004) consideran que los profesores pueden percibir las TIC de diferentes maneras, por ejemplo, les pueden tener miedo, pueden considerar que no son de utilidad para sus prácticas académicas, que no les ayudan a mejorar lo que ya hacen, que no les gusten o que sean renuentes a su uso, esto último probado en el estudio de Georgina y Olson (2007) -realizado en 15 universidades-, donde encontraron que el $75 \%$ de los profesores preferían dar clases en un aula sin integrar las TIC, mientras que solo un $25 \%$ estaba a favor de integrarlas.

En el estudio realizado por Alkubut (2001), se encontró que las percepciones de los profesores acerca de la tecnología, difieren respecto de los departamentos, género y la frecuencia de uso de las TIC para propósitos de instrucción; sin embargo, las percepciones no varían de acuerdo con los ingresos, la experiencia de uso de la computadora o si cuentan con computadora en el hogar.

Gibson (2008), por ejemplo, cuestiona a los profesores en relación con la facilidad de uso de las TIC; y su utilidad y efectividad en el contexto académico, esto último cuestionando al profesor si consideraba que las TIC son útiles en la educación, si hacen más efectiva la enseñanza y si las TIC son compatibles con su forma de enseñanza (p. 357). En lo anterior, coincide Kopcha (2012) quien asegura que las creencias 
del profesor universitario acerca de la utilidad y dificultad asociada con la integración de la tecnología, influye en si las llegan a usar o no para sus funciones académicas.

Por último, dicen Gulbahar y Guven (2008) que el profesor debe creer en la eficacia de la tecnología, que su uso no va causar disturbios en sus formas de enseñanza. Además, deben creer que ellos tienen el control sobre la tecnología. Otro punto de vista, es el de Jhonson et al. (2012) quienes afirman que la baja integración de las TIC por parte de los profesores se debe a que estos no entienden "por qué" deberían incorporar las TIC en sus clases, solo se les dice que necesitan utilizarlas pero no entienden por qué (p. 12).

\section{El profesor universitario y las TIC}

Las instituciones de Educación Superior buscan incorporar las TIC a sus prácticas académicas cotidianas, utilizando -en el mejor de los casos- directrices internacionales como los reportes anuales Horizonte y referentes de organismos como UNESCO, OCDE, ISTE, ILCE. Sin embargo, la implementación de estos lineamientos internacionales en contextos locales podría no ser pertinente por no considerar el sentido regional de la institución. En este sentido, Ramírez, Casillas, Morales y Olguín (2014) sugieren que es deseable una revisión y diagnóstico de las condiciones institucionales antes de implementar políticas y estrategias de TIC provenientes de organismos internacionales.

La integración de las TIC por parte del profesor universitario es un fenómeno complejo, pues primero que nada se asume que hay desigualdades de diferentes dimensiones y magnitudes llamadas brechas digitales (Ramírez, Casillas, Morales y Olguín, 2014), además de que como se menciona en el reporte Horizonte (2013), la mayoría de los académicos no están utilizando tecnologías que sean significativas para el aprendizaje y la enseñanza, ni para organizar sus investigaciones, es decir, que el impacto de las TIC es sumamente incierto, más aún como lo señala Alarcón y Ortiz (2013), quienes encontraron que no hay evidencia -en México- de que el uso e integración de las TIC en la Educación Superior, tenga un impacto positivo en los procesos académicos.

Ante la complejidad de la integración de las TIC, en esta investigación se considera que uno de los factores que se han estudiado poco en la literatura, es la diferenciación que hay de acuerdo con la disciplina de los 
profesores, ya que la disciplina enmarca diferencias en la universidad, formando distintas tribus como les denomina Becher (2001) o comunidades académicas según Clark (1991). Según Becher (2001) y Trowler (2012), estas tribus - o agrupaciones- poseen distintas culturas académicas, es decir, que tiene una variedad de formas simbólicas, conocimientos, prácticas, creencias e ideologías que les separa de las demás tribus.

En términos de Pierre Bourdieu (1979), los profesores -de acuerdo con su comunidad académica- poseen un habitus colectivo, es decir, un esquema de disposiciones -como creencias, ideales, miedos, valores, etc- construidos durante su historia, que emergen en su contexto (Guevara, 2010), -en este caso el académico-. Es así como se asume que los profesores construyen un habitus colectivo respecto de las TIC (disposiciones respecto a ellas), y que este emerge e impacta en las acciones para integrar las TIC a sus funciones académicas, pues como lo dice Guevara esto determinará -en términos individuales y grupales- si se integran o no las TIC.

\section{Marco contextual}

En el caso de la Universidad Veracruzana, es preciso revelar que es una institución que desde el año 2000 (UV, 2012) se ha propuesto ser una universidad tecnologizada y para esto ha hecho inversiones importantes y llevado a cabo estrategias de integración tecnológica, como la inversión que se realizó en equipamiento y conectividad; el diseño y conducción de proyectos que consideran como uno de sus ejes rectores a las TI, entre los cuales destacan el Proyecto Aula y el marco común de computación dentro del Modelo Educativo Integral y Flexible (MEIF); la oferta de programas de capacitación continua para los profesores-que incluye uso de tecnología-; y la provisión de servicios tecnológicos como el correo institucional, portal institucional para estudiantes y profesores, una plataforma educativa propia -desarrollada al interior de la institución- Eminus, iTunes-UV, la Red de Internet a lo largo de toda la Universidad (RIUV), salas de videoconferencia, entre otros muchos que la presentan como una universidad con una disposición tecnológica alta.

Es una universidad que cuenta con 6,013 profesores (UV, 2014) a lo largo de sus cinco regiones (Xalapa, Veracruz, Poza Rica-Tuxpan, Coatzacoalcos-Minatitlan, y Córdoba-Orizaba) y tiene presencia en 28 municipios del Estado. Xalapa es el campus donde 
se concentra la mayor cantidad de servicios, programas de estudio, estudiantes y profesores.

La licenciatura en Informática es un programa académico que se encuentra adscrito a la facultad de estadística e informática ubicada en la región Xalapa, que cuenta con 35 años de antigüedad, fue el primer programa a nivel regional a finales de los 70 lo que la posicionó como pionera en soluciones administrativas en el sector gubernamental y empresarial y es considerada como una de las facultades con mayor disposición y afinidad tecnológica. Esta cuenta con una planta académica de 65 profesores.

De los 65 que conforman la planta académica, se encontraban 62 activos en el periodo de enero-agosto, 2014 (FEI, 2014) y del total de profesores activos, se encuestaron 46 . Al respecto de ellos, podemos expresar que, el $70 \%$ tiene grado de maestría, $17 \%$ doctorado, $6 \%$ especialidad y $3 \%$ licenciatura; en términos de edad, el $15.2 \%$ son menores de 30 años, el $28.3 \%$ tienen entre 31 y 40 años, el $23.9 \%$ entre 41 y 50 años, el $26.1 \%$ entre 51 y 60 y únicamente $6.5 \%$ son mayores de 60 años, se puede observar que esta carrera tiene una tendencia a disponer de profesores jóvenes pues la media de edad es de 44 años y la mayoría de los profesores están por debajo de la media; con lo que respecta a género el $46 \%$ son hombres y el $56 \%$ mujeres, podemos desmitificar que esta licenciatura es exclusiva de un género.

\section{Metodología}

La metodología empleada en este proyecto se deriva de la investigación Brecha Digital-UV, en la que se ha planeado explorar el grado de apropiación tecnológica, el capital tecnológico y las trayectorias escolares -o desempeños académicos- de estudiantes y profesores universitarios, para lo cual se ha construido un instrumento para la recolección de datos que entre otros aspectos, permite indagar sobre las percepciones de estudiantes y profesores en relación con las TIC. Este instrumento cuenta con diez secciones que profundizan acerca de diferentes elementos de la integración tecnológica de los profesores, entre los cuales se encuentran: nivel socioeconómico; afinidad tecnológica; habilidad para manejar dispositivos, archivos, texto y texto enriquecido y contenido multimedia; comunicación, colaboración y socialización; por último literacidad digital y ciudadanía digital. 
En este artículo, por estar enfocado en la percepción de los profesores acerca de las TIC, se seleccionaron 17 indicadores del instrumento que provienen de dos secciones ( 5 de la sección de información socioeconómica y 12 de afinidad tecnológica), los cuales se utilizan para explorar sus creencias o percepciones respecto a las TIC (Véase Tabla 1). Los indicadores empleados han sido diseñados con un formato de escala likert cuyas opciones de respuesta son: Muy en desacuerdo (1); En desacuerdo (2); Indeciso (3); De acuerdo (4); Muy de acuerdo (5); y asimismo han sido validados a través de la prueba de Cronbach, utilizando el método de la varianza y el resultado fue de 0.86 .

Tabla 1. Indicadores de percepción de TIC, tomados del Proyecto de Brecha Digital, UV

\begin{tabular}{|l|l|}
\hline SOC 2.1 & Tener computadora, laptop, tableta o smartphone es símbolo de prestigio \\
\hline SOC 2.2 & Computadora o laptop es útil para actividades académicas \\
\hline SOC 2.3 & Tableta indispensable para útil académicas \\
\hline SOC 2.4 & Smartphone es útil para actividades académicas \\
\hline SOC 2.5 & Acceso a Internet útil para actividades académicas \\
\hline AFI 8.4 & Un celular con Internet es indispensable para vida cotidiana \\
\hline AFI 7.1 & Dominar tecnología a mejorar docencia \\
\hline AFI 7.2 & Dominar tecnología a mejorar gestión \\
\hline AFI 7.3 & Dominar tecnología a mejorar tutoría \\
\hline AFI 7.4 & Dominar tecnología a mejorar Investigación \\
\hline AFI 7.5 & Dominar tecnología a mejorar Vinculación \\
\hline AFI 8.2 & La mejor manera de encontrar información académica es en Internet \\
\hline AFI 8.3 & Indispensable mantenerse actualizado en software y hardware \\
\hline AFI 9.1 & $\begin{array}{l}\text { El Profesor que Domina TIC tiene mayor facilidad para establecer redes } \\
\text { académicas }\end{array}$ \\
\hline AFI 9.2 & Profesor domina TIC tiene mejores condiciones laborales \\
\hline AFI 9.3 & En el ámbito actual es imposible vivir sin TIC \\
\hline AFI 9.4 & Una publicación electrónica ofrece ventajas s/impresas \\
\hline
\end{tabular}

Los indicadores utilizados en conjunto revelan la percepción general de los profesores acerca de las TIC en el contexto académico, que a su vez revelan un primer acercamiento a un posible habitus colectivo respecto de las TIC. Es importante desmenuzarlo para comprender el porqué de estas creencias comunes -o no- acerca de las TIC en el contexto académico del profesor. 


\section{Resultados}

\section{Afinidad Tecnológica de los profesores}

Tomamos la definición de afinidad tecnológica del Proyecto Brecha Digital-UV para develar las percepciones de los profesores (la sumatoria de los 17 indicadores), que en este caso se han normalizado a 10.

Afinidad Tecnológica del Profesor Universitario $=(($ SOC $2.1+$ SOC $2.2+$ SOC $2.3+$ SOC $2.4+$ SOC $2.5+$ AFI $8.4+$ AFI $7.2+$ AFI $7.3+$

La media de la afinidad tecnológica de los profesores de informática es 7 (de un rango de $0-10$ ), y 28 profesores de 46 están por arriba de la media, lo que hace que nos indica que el $60 \%$ tiene una percepción positiva acerca de las TIC en el contexto académico y en estos profesores se observan las siguientes características: no hay diferencias en cuanto al género pues la mitad son hombres y la mitad mujeres; en cuanto al tipo de contratación, existe una ligera tendencia a hacia los profesores de asignatura pues dos terceras parte de los profesores con afinidad alta son de asignatura; y en cuanto a la edad si tienden a ser profesores jóvenes (por debajo de la media de edad), ya que el 75\% de ellos son menores de 44 años.

En cuanto a los profesores cuya afinidad se encuentra por debajo de la media, son un total de 18 , se concentran en una afinidad de seis aproximadamente, y solo un profesor cuenta con una afinidad baja (4.24). Respecto de ellos, podemos expresar que: dos tercios de los profesores son mujeres; poco más de la mitad son profesores de tiempo completo; y al contrario de los de afinidad alta, estos profesores tienden a ser mayores, pues más del $70 \%$ son mayores de 48 años de edad. 
Figura 1. Afinidad tecnológica de los profesores de Informática

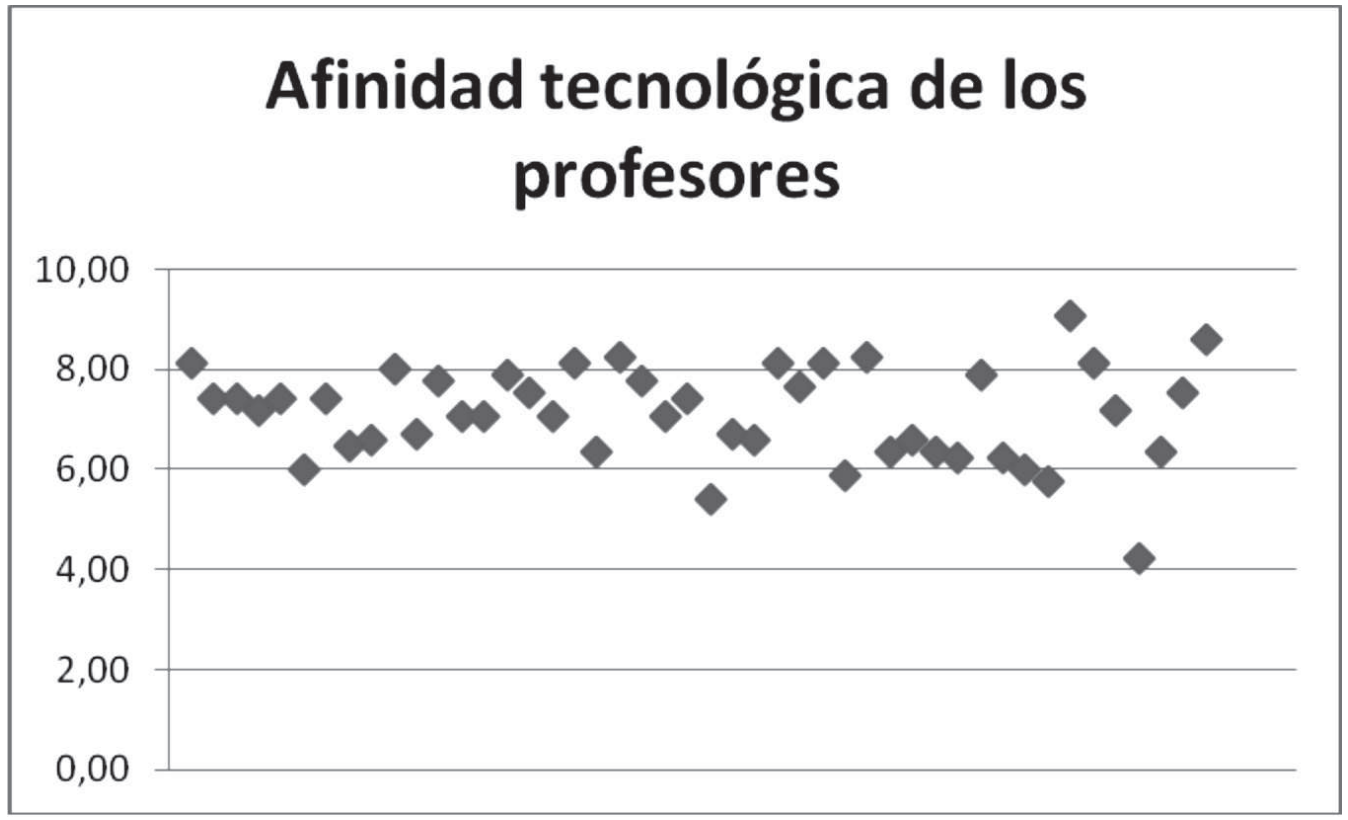

Fuente: Elaboración propia

Como se puede observar en la figura 1, la afinidad de los profesores permanece alrededor de la media (7) y el promedio (7.11) lo que permite concluir que en común cuentan con una percepción media-alta, aunque no determinista como para asegurar que las TIC tengan una utilidad absoluta y un impacto completamente positivo, aún cuando nuestro supuesto fue que los profesores de esta facultad son muy afines a la tecnología (pro-tecnológicos), y que inclusive se trataba de profesores deterministas respecto del uso de las TIC en la educación. Entendiendo como determinista tecnológico, (para este contexto y en términos de lo examinado), a un profesor que esté de acuerdo y muy de acuerdo con aseveraciones dentro del contexto académico:

- La computadora o laptop es indispensable para sus actividades;

- $\quad$ las tabletas y smartphones son útiles e indispensables;

- $\quad$ poseer dispositivos digitales (computadora, laptop, tableta y smartphone) es símbolo de prestigio;

- $\quad$ el acceso a internet es indispensable;

- $\quad$ el manejo de las TIC mejora sus funciones académicas (investigación, docencia, gestión, vinculación y tutoría); 
- $\quad$ el profesor que maneja las TIC tiene mayor facilidad para establecer redes académicas;

- $\quad$ el profesor que domina las TIC puede mejorar sus condiciones académicas;

- la mejor manera de encontrar información es a través de Internet;

- $\quad$ es indispensable mantenerse actualizado en software y hardware;

- $\quad$ que una publicación electrónica ofrece mayores ventajas sobre las impresas;

- $\quad$ y por último en el ámbito actual, es imposible vivir sin las TIC.

Todo lo anterior, expresa las características de lo que en este trabajo de investigación, entendemos por un profesor determinista tecnológico. De acuerdo con esta situación, lo mostrado en la figura 1, hemos descubierto que los profesores que se encuentran entre los valores 8 y 10 son deterministas tecnológicos pues quienes tienen una afinidad tecnológica dentro de este rango, están totalmente de acuerdo y muy de acuerdo con todas las aseveraciones antes mencionadas. Siendo así, se pensaría que los profesores de la licenciatura en Informática, al ser las TIC su objeto de estudio, serían deterministas tecnológicos, sin embargo solo se cuenta con diez profesores cuya percepción corresponde a lo que identificamos como determinista tecnológicos, de los cuales seis son hombres y cuatro mujeres; cinco de tiempo completo y cinco de asignatura; y la mayoría (8) son profesores jóvenes -debajo de 44 años-, y solo dos son mayores a 50 años (52 y 54).

Respecto del grueso de la población, cuentan con una afinidad alrededor del promedio de afinidad tecnológica, lo que nos permite observar que cerca del $80 \%$ de los profesores cuentan con una afinidad positiva pero no refieren a las TIC impactos fuertes en relación con lo cuestionado, lo que provoca, la revisión de las percepciones particulares de los profesores para indagar acerca de cuáles creencias hacen que no sean deterministas tecnológicos. Para esto se revisaron todos los indicadores (17), buscando aquellos en que los profesores declaran una percepción baja. Se encontraron ocho indicadores que alejan al profesor de tener una afinidad totalmente positiva (determinista) acerca del uso de las TIC en el contexto académico. Se separaron en tres rubros: a) Las TIC como símbolo de prestigio en el contexto académico; b) el uso de dispositivos móviles en el contexto académico; y c) la confiabilidad de fuentes de información y publicaciones en línea. 


\section{a) El impacto de las TIC en el contexto académico}

El 76\% de los profesores consideran que las TIC no son imprescindibles en el contexto académico, aún cuando son su objeto de estudio. Otro punto interesante es que casi la mitad (48\%) de los profesores no ven en las TIC una oportunidad de mejorar sus condiciones laborales, mediante el reconocimiento institucional (PEDPA) y nacional (PROMEP y SNI) de su desempeño académico.

Ahora bien, respecto del prestigio que da el contar con dispositivos digitales, ningún profesor declaró estar muy de acuerdo con esto, sólo cinco estuvieron de acuerdo, otros siete se declararon indecisos y la mayoría (34) están en desacuerdo y muy en desacuerdo con que poseer dispositivos digitales sea símbolo de prestigio, lo cual nos revela ese sentido pragmático que le atribuyen al uso de las TIC, pues no son para ellos solo cuestión de prestigio, sino que debido a la naturaleza de su disciplina ven en ellos un capital que bien canalizado se traducirá en éxito académico y reconocimiento a su desempeño.

\section{b) El uso de dispositivos móviles en el contexto académico}

Es revelador encontrar que los profesores de Informática tienen una percepción baja acerca del uso de dispositivos móviles (Smartphone y Tableta) en el contexto académico. Además, únicamente el 22\% está de acuerdo con que pueden llegar a ser útiles en el desarrollo de las funciones académicas, el $24 \%$ se declaran indecisos y el $54 \%$ están en desacuerdo y muy en desacuerdo con que sean indispensables. Y respecto de los Smartphone, el 28\% creen que si puede ser útil, el 17\% se declara indeciso y el $48 \%$ dicen estar en desacuerdo y muy en desacuerdo con que los smartphone sean útiles en el contexto académico. Lo anterior nos indica que aproximadamente la mitad de los profesores aún no vislumbran una utilidad del smartphone y la tableta en realizar sus funciones sustantivas, algunos aún se encuentran indecisos al respecto y son pocos los que empiezan a ver en ellos usos académicos. Por el contrario, la computadora y laptop todos los profesores las consideran un dispositivo tecnológico indispensable.

Adicionalmente, se encontró que una tercera parte de los profesores cuentan con tableta; sin embargo, no aclaran si es para uso académico o entretenimiento, aunque vale la pena mencionar que la facultad cuenta con tres tabletas a disposición de los desarrolladores de aplicaciones móviles, sean profesores o estudiantes. Para el caso del 
smartphone -cuya intención de uso también desconocemos- se encontró que casi el 70\%de los profesores cuentan con uno.

c) La confiabilidad de fuentes de información y publicaciones en línea.

En relación con la confiabilidad de fuentes de información electrónicas (publicaciones en línea), la percepción de los profesores está dividida pues el $52 \%$ le confiere a Internet altos niveles de confiabilidad, mientras que el $48 \%$ o se declara indeciso o no está de acuerdo. En este sentido, podríamos expresar varias hipótesis que tratarán de explicar esta situación; no obstante, no se cuenta con elementos suficientes para entender las razones de la confianza -o falta de ella- de Internet como una fuente de información académica veraz, en este mismo sentido el $58 \%$ de los profesores si reconoce ventajas de las publicación en línea sobre las impresas.

\section{Afinidades tecnológicas comunes entre los profesores de informática}

Es preciso resaltar que las percepciones -disposiciones, creencias comunes- que hacen que los profesores de Informática, sean afines a la tecnología, son: El acceso a recursos tecnológicos como la computadora y conectividad a Internet; y el impacto que consideran podrían tener las TIC en sus funciones académicas.

\section{1) Impacto de las TIC en sus funciones académicas}

En la literatura se presume que la percepción del profesor acerca de las TIC, es la principal determinante para la incorporación efectiva de las TIC, y resulta importante ver su percepción de impacto en cada una de sus funciones académicas: docencia, investigación, gestión, vinculación y tutoría, las cuales son declaradas como las funciones sustantivas de un profesor universitario (UV, 2012).

De manera general, los profesores comparten la idea de que las TIC sí tienen impacto positivo en todas sus funciones académicas y cabe señalar que la investigación es la función en que los profesores consideran que las TIC pueden tener mayor impacto ( $89 \%$ de acuerdo y muy de acuerdo), en segundo lugar se encuentra la docencia (87\%), gestión con un $83 \%$, vinculación con un $80 \%$ y la función en la que menos creen que las TIC pueden ser de utilidad o que pueden tener impacto es en la tutoría. 
Por otro lado, existe la creencia casi absoluta (95.7\%) de que un aspecto del contexto universitario que puede verse beneficiado por el uso de las TIC, es la comunicación entre colegas y estudiantes, pues repercute en dos aspectos muy importantes: el establecimiento de redes de colaboración mediante las TIC y el intercambio de información y comunicación con los estudiantes fuera del aula.

\section{2) Acceso a recursos tecnológicos (Computadora e Internet)}

Una idea generalizada entre los profesores radica en que la computadora y el Internet son indispensables en el contexto académico, lo que devela a estos como los recursos tecnológicos que se perciben con mayor importancia en su contexto.

El 100\% de los profesores coincide en que el uso de la computadora de escritorio o laptop es indispensable para realizar sus actividades académicas y como era de esperarse todos los profesores cuentan con una computadora personal (de escritorio o laptop).

En cuanto al acceso a Internet, el 100\% de los profesores están de acuerdo y muy de acuerdo en que permanecer conectado a internet es indispensable para sus funciones académicas, aunado a esto se encontró que 41 de 46 profesores cuentan con servicio de Internet en casa.

\section{Conclusión}

De acuerdo con lo expresado por Gulbahar y Guven (2008); Georgina y Olson (2007); Zhoa y Cziko (2001), la percepción de las TIC por parte de académicos universitarios ha sido reconocida como un factor de vital importancia para el éxito de la integración tecnológica dentro de la educación. En este artículo, se estudiaron las percepciones de los profesores que bajo el supuesto de que contarían con una afinidad tecnológica muy alta y que serían deterministas tecnológicos, sin embargo, se encontró que la comunidad académica de la facultad de informática está compuesta por profesores que si bien han construido afinidades comunes -o un habitus colectivo en términos de Bourdieu-, no son altamente afines al uso de las TIC en el contexto universitario, sino que cuentan con una afinidad entre media y alta.

Es decir, que si cuentan con afinidades comunes, pero hay ciertos elementos acerca del uso de las TIC, en los que tienen sus reservas que a su vez los alejan de ser deterministas tecnológicos; empero es revelador 
encontrar que uno de los elementos que alejan a los profesores de ser altamente afines con las TIC, es el uso de dispositivos móviles -tabletas y smartphones- dado que a pesar de ser señalados como tecnologías de alto impacto en la Educación Superior en los reportes Horizonte (Jhonson, et. al., 2013), los profesores de esta facultad no los reconocen como tal; por lo cual esta situación es el primer indicio de una baja penetración de estos dispositivos en el ámbito en que se desenvuelven los profesores, pues como lo indica Zare-ee (2007) si la percepción es limitada, el uso por ende será limitado.

Es contradictorio que uno de los aspectos que los profesores creen que puede verse beneficiado por el uso de las TIC, es la comunicación, sin embargo nuestros datos no reflejan que sean tecnologías a las que se les valore de forma positiva para ser usadas en el ámbito académico, aún sabiendo que pueden favorecer de manera significativa la comunicación entre estudiantes y profesores.

Otro punto que vale la pena señalar, es que una tercera parte de los profesores que consideran que las TIC no son imprescindibles, son profesores cuyo objeto de estudio son precisamente las TIC. Si creen que Internet es una fuente de información importante, pero existen dudas sobre su grado de confiabilidad.

Ahora bien, es preciso resaltar que la afinidad tecnológica común - habitus, creencias, disposiciones, ideologías de TIC- de los profesores estudiados se caracteriza por dos aspectos: 1) que ellos creen que las TIC son un factor de impacto positivo para sus funciones académicas -siendo la investigación a la que asumen mayores beneficios, seguida de la docencia, la gestión, vinculación y tutoría- y 2) que todos los profesores encuestados creen que los recursos indispensables dentro del contexto académico se refieren a la computadora o laptop y al acceso a Internet. Con esto se puede observar que los profesores son afines a las TIC pero no significa que crean que éstas sean en todo momento símbolo de prosperidad, modernidad y bonanza dentro del ámbito académico.

Finalmente, es importante expresar que este es el primer acercamiento que tenemos -en la investigación de Brecha Digital en Educación Superior-a la interpretación de datos que ayudan a reconocer a los profesores universitarios como sujetos que incorporan o no a las TIC a sus prácticas académicas. De este estudio, se derivarán futuras comparaciones que abonen al entendimiento de la afinidad tecnológica como elemento que se construye al interior de cada comunidad disciplinaria; 
asimismo se resalta la importancia de estudiarlo a profundidad con métodos que permitan comprender a la percepción de las TIC como un fenómeno complejo y subjetivo pero necesario para el análisis de la integración de las TIC en educación superior. Es necesario continuar con las exploraciones de este fenómeno, pero bajo métodos cualitativos pues en este artículo se sientan las bases para realizarlo pero de forma limitada en cuanto al acercamiento y profundidad debido al avance de la investigación y a la intención del artículo.

\section{Referencias bibliográficas}

Alarcón E. y Ortiz V. (2013). Estudiantes, profesores y TIC: La investigación en México. En Ramírez- Martinell A. y Casillas A.M., Háblame de TIC: Tecnología Digital en la Educación Superior. 1a ed. Córdoba: Editorial Brujas.

Akbulut, Y., Odabasi, H., \& Kuzu, A. (2011). Perceptions of preservice teachers regarding the integration of information and communication technologies in Turkish education faculties. Turkish Online Journal of Educational Technology - TOJET, 10(3), 175-184.

Bourdieu, P. (1987). Los tres estados del capital cultural. Sociológica, UAM,11-17.

Castells, M. (2002). La era de la información: Economía sociedad y cultura. La sociedad red (Cuarta ed., Vol. I). Distrito Federal, México: Siglo XXI Editores.

Clark B. (1991). El Sistema de Educación Superior: Una visión comparativa de la organización académica. Editorial Nueva Imagen/ Universidad.

Georgina, D. A., \& Olson, M. R. (2008). Integration of technology in higher education: A review of faculty self-perceptions. Internet and Higher Education, 11(1), 1-8.

Georgina, D. A., \& Hosford, C. C. (2009). Higher education faculty perceptions on technology integration and training. Teaching and Teacher Education: An International Journal of Research and Studies, 25(5), 690-696.

Gulbahar, Y., \& Guven, I. (2008). A survey on ICT usage and the perceptions of social studies teachers in turkey. Educational Technology and Society, 11(3), 37-51. 
Guevara, C. H. (2010). Integración tecnológica del profesor universitario desde la teoría social del Pierre Bourdieu. (U. d. Guadalajara, Ed.) Apertura Electrónica, (Número especial), 7.

Johnson, T., Wisniewski, M., Kuhlemeyer, G., Isaacs, G., \& Krzykowski, J. (2012). Technology adoption in higher education: Overcoming anxiety through faculty bootcamp. Journal of Asynchronous Learning Networks, 16(2), 63-72.

Johnson, L., Adams Becker, S., Gago, D. Garcia, E., y Martín, S. (2013). NMC Perspectivas Tecnológicas: Educación Superior en América Latina 2013-2018. Un Análisis Regional del Informe Horizonte del NMC. Austin, Texas: The New Media Consortium.

Kopcha, T. J. (2012). Teachers' perceptions of the barriers to technology integration and practices with technology under situated professional development. Computers and Education, 59(4), 1109-1121.

Ouzts, D. T., \& Palombo, M. J. (2004). Technology in higher education: A study of perceptions of college professors. Tech trends: Linking Research and Practice to Improve Learning, 48(5), 19-24, 84.

PETIC-UV. (2012). Plan Estratégico de Tecnologías de Información y Comunicaciones, Dirección General de Tecnologías de Información. Recuperado el 15 de Abril, desde http://www.uv.mx/transparencia/files/2012/10/PlandeDesarrolloTecnologicoUV.pdf

Ramírez Martinell, A. (2012). Saberes digitales mínimos en Centros de Auto Acceso. Presentación Plenaria en el Encuentro Internacional de Centros de Auto Acceso. Universidad Veracruzana, Xalapa, Ver.

Ramírez M. A., Morales R.A., Olguín A. P. (2012). Marcos de Referencia de Saberes Digitales. En proceso de publicación, 1-26.

Trowler, P., Knight P. (2000) Departament-level Cultures and the Improvement of Learning and Teaching. Studies in Higher Education. Volume 25, No. 1, Carfax Publishing.

Universidad Veracruzana. (2012). Universidad Veracruzana. Recuperado de http://www.uv.mx/

Universidad Veracruzana (2014). Información Estadística, UV. Recuperado de http://www.uv.mx/informacion-estadistica/consultas-dinamicas/

Zare-ee, A. (2011).University teachers' views on the use of information communication technologies in teaching and research. Turkish Online Journal of Educational Technology-TOJET, 10(3), 318-327. 